\title{
The effects of lesions in the ventromedial nucleus of the hypothalamus on behavioral contrast in rats*
}

\author{
MICHAEL L. JAFFE \\ Adelphi University, Garden City, New York 11530
}

\begin{abstract}
Rats with lesions in the ventromedial nucleus of the hypothalamus displayed significantly greater positive behavioral contrast effects than did controls on a multiple VI extinction schedule, supporting the hypothesis that animals with ventromedial lesions are more responsive than controls to changes in reinforcement contingencies. Also supporting this hypothesis was the finding that three of the four lesioned animals displayed negative behavioral contrast effects on a multiple VI VI schedule which followed the multiple VI extinction schedule, whereas none of the five control animals displayed negative contrast. Manipulations involving shifts in reinforcer quality were inconclusive. It was concluded that some of the current hypotheses regarding the behavioral consequences of ventromedial lesions are in need of revision.
\end{abstract}

Several studies have demonstrated that animals with ventromedial hypothalamic (VMH) lesions show greater reactivity to the positively reinforcing and aversive properties of stimuli than do control animals. For example, the occurrence of overeating normally seen in VMH-lesioned rats critically depends upon the taste qualities of the diet which the animals are fed (Corbit \& Stellar, 1964; Graff \& Stellar, 1962; Teitelbaum, 1955). While normal animals will consume appreciable amounts of cellulose- or quinine-adulterated food, obese hyperphagic rats will stop eating and lose a great deal of weight (Graff \& Stellar, 1962). Obese hyperphagic animals will also show a marked increase in food intake over their own control levels if their food is sweetened with dextrose (Teitelbaum, 1957).

Other studies have corroborated these findings for animals in either the dynamic or static phases of hyperphagia, showing that they react exaggeratedly to the taste qualities of both food (Corbit \& Stellar, 1964) and water (Corbit, 1965). Furthermore, intragastric feeding studies have demonstrated that although unoperated rats can regulate food and water intake in the absence of taste and smell, VMH-lesioned animals are critically affected by the absence of taste (Epstein \& Teitelbaum, 1962; Teitelbaum \& Epstein, 1963).

The exaggerated finickiness shown by animals with the VMH area destroyed has been used to support the thesis that VMH lesions produce a basic motivational disturbance evidenced by increased sensitivity to the reinforcing properties of sensory stimulation. According to Grossman $(1966,1967)$, the effects of VMH lesions on feeding behavior may be secondary to a more general change in the organism's affective responsiveness to all stimuli.

In addition, it has been demonstrated that rats with lesions in the VMH nucleus do not perform as well as control animals on a wide variety of appetitive

*This study is based on the author's $\mathrm{PhD}$ dissertation, completed at Adelphi University, 1972, and supervised by $M$. Caplan. A shorter version of this paper was presented at the annual meeting of the Eastern Psychological Association, 1973. instrumental tasks (Grossman, 1966; Miller, Bailey, \& Stevenson, 1950; Teitelbaum, 1957; Sclafani, 1971). The reported changes in reactivity to reinforcement contingencies are not restricted to appetitive instrumental tasks. It has been shown that both rats (Miller et al, 1950; Turner, Sechzer, \& Liebelt, 1967) and mice (Turner et al, 1967) with VMH lesions demonstrate lower shock thresholds than do control animals, and that hyperphagic mice require less than half the number of trials to reach criterion on a shock avoidance task compared to normal controls (Sechzer, Turner, \& Liebelt, 1966). Grossman (1966) found that VMH-lesioned rats performed better than controls on an active avoidance task. These studies indicate that a given shock level is more aversive to VMH-lesioned animals than to controls.

The studies cited above suggest that VMH lesions alter an animal's responsiveness to stimuli and to reinforcement contingencies. The fact that VMH-lesioned animals eat less adulterated food but more palatable food than normals demonstrates an exaggerated responsiveness to the taste properties of these stimuli. The findings involving the "poorer" performance of VMH-lesioned animals on instrumental tasks also support this general analysis insofar as the contingencies of reinforcement operating in these tasks may become aversive to the animal (e.g., Teitelbaum, 1957). In addition, as previously cited, VMH-lesioned animals have been shown to have lowered shock thresholds, and they have performed better than controls on active avoidance tasks. These findings also are indicative of the exaggerated responsiveness of VMH-lesioned animals to stimuli.

Jaffe and Caplan (1971) tested the hypothesis that VMH lesions result in increased responsiveness to changes in reinforcement contingencies by examining changes in response rates resulting from switching the contingencies of two reinforcement schedules. It was assumed that the incentive contrast paradigm was sensitive to behavioral changes resulting from manipulations involving the relative reinforcing 
properties of schedules of reinforcement. Both normal and VMH-lesioned rats were trained on either a fixed-interval (FI) 30-sec or FI 150-sec schedule of food reinforcement. When stable rates of responding were established, the animals on FI $30 \mathrm{sec}$ were switched to FI $150 \mathrm{sec}$, and the animals on FI 150 were switched to FI 30. The lesioned animals demonstrated marked positive contrast in response to an increase in reinforcement density, as well as marked negative contrast when reinforcement density was reduced. The control animals did not display corresponding contrast effects.

Since the results of the above experiment were consistent with the general hypothesis that VMH-lesioned animals exhibit greater responsiveness to changes in reinforcing properties of stimulus events than do normal animals, the authors concluded that the most parsimonious explanation for the diverse effects that have been obtained subsequent to VMH lesions is one that posits a general change in the organism's responsiveness to changes in the reinforcing properties of stimulus events.

The above experiment utilized a between-S incentive contrast paradigm. The behavioral contrast paradigm is defined on the basis of within-S comparisons between pre- and postshift response rates displayed by the same animal, with the S's preshift baseline performance serving as a control rate with which subsequent rate changes are compared (Dunham, 1968). A preshift baseline of responding exists for each animal, permitting the assessment of both degree and direction of contrast effects. The amount of contrast can be used to gauge differences in responsiveness to changes in the reinforcing properties of schedules used between the experimental and control animals.

The rationale for the present experiment is based upon the following assumptions, supported by previous experimentation: (1) that VMH-lesioned animals are more responsive than controls to increases in the positive reinforcing properties of stimulus events insofar as they will evidence greater approach, consummatory, or rate behaviors; (2) that VMH-lesioned animals are more responsive than controls to increases in the aversive properties of stimulus events insofar as they will evidence greater withdrawal behaviors or decreases in rate or probability of response; and (3) that the behavioral contrast paradigm is a procedure that permits an evaluation of responsiveness to changes in the reinforcing properties of stimulus events, and therefore will allow comparisons of the relative aversiveness of these events for VMH-lesioned and control animals.

The prediction made, then, is that animals with lesions in the ventromedial nucleus will show both greater positive and negative behavioral contrast than controls, resulting from manipulations of reinforcer density and quality on multiple reinforcement schedules. Positive behavioral contrast is defined as an increase in response rate above preshift baseline levels in the unchanged component of the multiple schedule. Negative contrast is defined as a decrease in response rate below that of the preshift baseline level in the unchanged component of the multiple schedule.

\section{METHOD}

\section{Subjects}

The Ss were nine male albino rats of the Charles River CD strain, approximately 90 days of age at the time of surgery. Four Ss received bilateral ventromedial hypothalamic lesions, four Ss served as normal controls, and one additional control animal received a sham operation. The Ss were individually housed and given ad lib access to water throughout the experiment. During the course of the study, Ss were maintained at approximately $80 \%$ of their free-feeding body weights. Approximately half-way through the experiment, the $80 \%$ weight levels were adjusted to minimize the effects of increasing deprivation due to normal growth.

\section{Apparatus}

The apparatus consisted of a standard operant conditioning chamber (Scientific Prototype), $10 \mathrm{in.} \mathrm{long,} 10 \mathrm{in.} \mathrm{high,} \mathrm{and}$ $6 \frac{1}{2}$ in. wide, with a bar located 1 in. to the right of the feeder tray. An audible click from the feeder accompanied each reinforced response. During the phases of the experiment involving shifts in reinforcer quality, two feeders were connected to the feeder tray via a common rubber tube. Regular reinforcers consisted of standard Noyes food pellets $(45 \mathrm{mg})$; the adulterated food pellets were similar to the regular pellets except that they contained $.005 \%$ quinine (with concomitant changes in brightness, odor, and taste). The high-quality reinforcers consisted of Noyes sucrose pellets $(45 \mathrm{mg})$.

Programming of reinforcement contingencies and the recording of experimental data were accomplished by using BRS solid-state modules. A printout counter recorded the number of responses and the number of reinforcements per minute. During the fifth phase of the experiment, a module was added to the program to prevent the reinforcement of barholding behavior.

\section{Operations and Histology}

Prior to surgery, experimental animals were shaped to barpress with food reinforcement and were then run on a VI $30-\mathrm{sec}$ schedule for approximately 12 days (that is, until daily response rates indicated stabilization).

Following rate stabilization, bilateral VMH lesions were stereotactically produced with a stainless steel electrode, insulated except for $.5 \mathrm{~mm}$ at the tip. All operations were performed with Nembutol anesthesia $(44 \mathrm{mg} / \mathrm{kg})$. The stereotactic coordinates employed for the lesions were $5.8 \mathrm{~mm}$ posterior to bregma, $0.7 \mathrm{~mm}$ lateral to the midline, and $9.4 \mathrm{~mm}$ below the surface of the cortex (deGroot, 1959). The lesions were produced by anodal currents of $2 \mathrm{~mA}$ delivered for $15 \mathrm{sec}$. One control animal received a sham operation, identical to the experimental operation except that electrodes were not inserted into the brain.

At the conclusion of the experiment, the lesioned animals were sacrificed, perfused intracardially with saline, and fixed with Formalin; the brains were then removed. During histology, the brains were sectioned at 40-micron intervals and stained with cresyl violet.

\section{Procedure}

Following random assignment of Ss to groups, the animals were trained on a VI 30 -sec schedule of food reinforcement, 
Table 1

Summary of Experimental Treatments

\begin{tabular}{|c|c|c|c|c|c|c|c|c|c|c|c|}
\hline \multirow[b]{3}{*}{ Phase } & \multirow[b]{2}{*}{ Schedule and $S$} & & \multicolumn{9}{|c|}{ Number of Sessions } \\
\hline & & nulus Conditions & \multicolumn{9}{|c|}{ Subject } \\
\hline & S1 & S2 & 25 & 27 & 28 & 29 & 33 & 34 & 35 & 36 & 37 \\
\hline 1 & \multicolumn{2}{|c|}{ VI $30 \mathrm{Sec}$ (Light On) } & 21 & 26 & 11 & 36 & 20 & 10 & 32 & 19 & 25 \\
\hline 2 & Ext (Off) & VI $30 \mathrm{Sec}(\mathrm{On})$ & 10 & 10 & 12 & 10 & 6 & 10 & 6 & 10 & 10 \\
\hline 3 & VI $30 \mathrm{Sec}$ (Off) & VI $30 \mathrm{Sec}(\mathrm{On})$ & 16 & 10 & 10 & 13 & 7 & 12 & 9 & 8 & 8 \\
\hline 4 & VI $30 \mathrm{Sec}^{*}$ (On) & VI $30 \mathrm{Sec}$ (Off) & 10 & 10 & - & 12 & 10 & 10 & 10 & 10 & 10 \\
\hline 5 & VI $30 \mathrm{Sec}$ (Off) & VI $30 \mathrm{Sec}(\mathrm{On})$ & 12 & 18 & - & 20 & 19 & 21 & 9 & 15 & 19 \\
\hline 6 & VI 30 Sect (On) & VI $30 \mathrm{Sec}$ (Off) & 4 & 6 & - & - & 6 & 6 & 6 & 6 & 6 \\
\hline
\end{tabular}

with the houselight on for the entire session (single stimulus training). This constituted Phase 1 of the experiment. For all phases, Ss were run approximately 7 days a week, each daily session lasting $50 \mathrm{~min}$ and consisting of alternating 5-min components (with the exception of Phase 1) on a multiple schedule. In Phases 2 through 6, the on and off states of the houselight served as discriminative stimuli for the components of the multiple schedule. Thus, a daily session consisted of 10 alternating 5-min components, 5 min with Schedule 1 in effect followed by $5 \mathrm{~min}$ with Schedule 2 in effect.

Following rate stabilization in Phase 1 (consisting of at least 5 consecutive days of no significant change in response rate as judged by visual inspection, with the exception of Animal 27, who was shifted after 4 consecutive days of no significant change), the experimental animals underwent surgical operations. Following a short recovery period (ranging from 3 to 9 days), they were returned to the VI $30-\mathrm{sec}$ schedule to ensure rate stabilization prior to Phase 2 and to permit observation of the effect of the operation on baseline response rate. This procedure was also followed for the sham-operated control animal (No. 35). The four additional control animals were not run during the surgical recovery period to ensure equivalent baseline training experiences for the two groups.

Phase 2 for all Ss consisted of a multiple VI 30-sec extinction schedule (mult VI ext) of reinforcement, with the VI 30-sec component being associated with light-on (for all Ss) and extinction being associated with light-off. Each session began with the extinction component and ended with the VI component, with strict alternation of schedules. Ss were run for approximately 10 days in Phase 2, with two control Ss being run for only 6 days each. During Phases 3 through 6 , a mult VI 30 VI 30-sec schedule of reinforcement was associated with various manipulations involving the quality of the reinforcer. Phases 3 and 5 served as restabilization periods following the manipulations of reinforcer density and quality in Phases 2 and 4. Phases 3 and 5 each consisted of the mult VI 30 VI $30-\mathrm{sec}$ schedule, with regular food pellets serving as reinforcers in both components.

In Phase 4 , food pellets adulterated with $.005 \%$ quinine were used as reinforcers when the houselight was on, whereas regular pellets served as reinforcers for the light-off component. In Phase 6, sucrose food pellets were used as reinforcers when the houselight was on, and regular food pellets served as reinforcers when the houselight was off. Table 1 summarizes the experimental manipulations for the six phases of the experiment.

\section{RESULTS}

\section{Anatomical Results}

Histological analysis revealed that all lesions damaged the ventromedial nucleus bilaterally as well as portions of the anterior hypothalamic nucleus (Ss 25, 27, and 36 ), the dorsomedial nucleus (Ss 27, 29, and 36), and arcuate nucleus (all Ss), the lateral nucleus (S 25), the paraventricular nucleus (all Ss), the supraoptic nucleus (S 27), and the fornix (S 25).

Extensive bilateral damage to the ventromedial nucleus occurred in all animals except S 25, who displayed extensive unilateral damage in the left side of the VMH and partial damage to the right side. All lesions extended from the A 4110 to the A 6280 planes of the Konig and Klippel (1963) atlas of the rat brain. The lesions were very similar to those found in previous studies in animals displaying the so-called "VMH syndrome" (Jaffe \& Caplan, 1971).

\section{Behavioral Data}

\section{Pre-and Postoperative Rates}

There were no significant changes in the Phase 1 (VI $30-\mathrm{sec}$ ) rates of response for the VMH-lesioned animals as a result of the lesion, although a temporary depression in rate occurred in three of the four lesioned animals and in the sham-operated control animal following the operation. The preoperative mean response rate for the four experimental animals averaged over the three sessions preceding the operation was 22.8 responses, compared to a mean postrecovery baseline rate of 23.5 responses, computed for the last 5 days preceding Phase 2. There was no change in response rate for the sham-operated control animal (No. 35), whose data are included with the data of the other control animals.

\section{Reinforcement Density Shift}

Table 2 contains a summary of the results for the Phase 2 mult VI 30 ext schedule, including the Phase 1 preshift baseline rate of response (calculated by averaging the rates of response for the five sessions preceding the shift) and the mean percent change in response rate averaged over sessions for each animal in Phase 2. Mean percent change was calculated by subtracting the preshift baseline rate from the mean 
Table 2

Summary of Results for Phase 2 (Mult VI Ext)

\begin{tabular}{|c|c|c|c|}
\hline$S$ & Condition & $\begin{array}{c}\text { Preshift } \\
\text { Baseline } \\
\text { (Responses/ } \\
\text { Minute) }\end{array}$ & $\begin{array}{c}\text { Postshif } \\
\text { Percent } \\
\text { Change } \\
\text { in Rate } \\
\text { in VI } \\
\text { Comp }\end{array}$ \\
\hline $\begin{array}{l}28 \\
33 \\
34 \\
35 \\
37\end{array}$ & $\begin{array}{l}\mathrm{C} \\
\mathrm{C} \\
\mathrm{C} \\
\mathrm{C} \\
\mathrm{C}\end{array}$ & $\begin{array}{r}20.2 \\
20.0 \\
16.0 \\
32.4 \\
19.9 \\
\bar{X}=21.7\end{array}$ & $\begin{array}{r}-3.5 \\
-0.5 \\
+33.8 \\
-6.7 \\
+2.2 \\
\bar{X}=+5.6\end{array}$ \\
\hline $\begin{array}{l}25 \\
27 \\
29 \\
36\end{array}$ & $\begin{array}{l}\text { VMH } \\
\text { VMH } \\
\text { VMH } \\
\text { VMH }\end{array}$ & $\begin{array}{r}27.2 \\
20.4 \\
24.7 \\
21.6 \\
\overline{\mathrm{X}}=23.5\end{array}$ & $\begin{aligned} & +33.9 \\
& +28.0 \\
& +98.5 \\
& +29.6 \\
\overline{\mathrm{X}}= & +47.5\end{aligned}$ \\
\hline
\end{tabular}

postshift rate, dividing by the preshift baseline rate, and then multiplying by 100 . The mean preshift baseline rate for the experimental group was 23.5 responses and for the control group was 21.7 responses. The two means did not differ significantly $[\mathrm{U}(4,5)=4.0, \mathrm{p}>.05$; Mann-Whitney U test was used for all statistical comparisons].

The mean percent change in rate for the experimental group in the unchanged VI component in Phase 2 (mult VI ext) was $+47.5 \%$ and for the control group was $+5.6 \%$. The difference between means was significant $[\mathrm{U}(4,5)=2, \mathrm{p}<.05]$.

Figure 1 contains the group data for both groups for Phases 1 through 6 . The figure shows the last five preshift sessions in Phase 1 (VI $30 \mathrm{sec}$ ), the mult VI ext

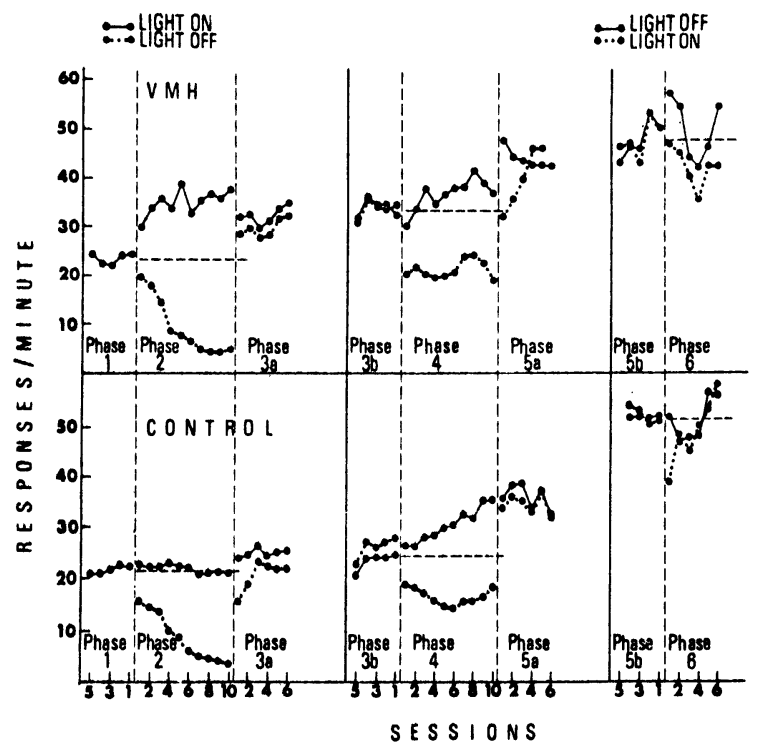

Fig. 1. Mean response rates for VMH-lesioned group (upper graph) and control group (lower graph) for Phases 1 through 6. In all figures, baseline rates for the unchanged components are indicated by the dashed horizontal lines. See Table 1 for schedules associated with light-on and light-off states.

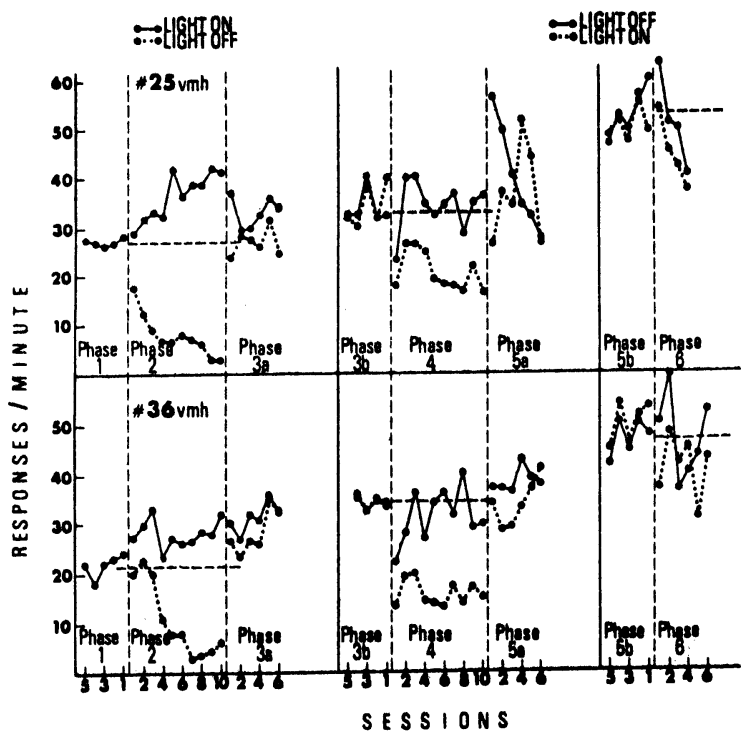

Fig. 2. Response rates for VMH-lesioned Animals 25 (upper graph) and 36 (lower graph) for Phases 1 through 6.

schedule rates in Phase 2, the first six (Phase 3a) and last five (Phase 3b) session rates in Phase 3 (mult VI 30 VI 30), the mult VI 30 VI 30 -sec schedule rates in Phase 4 with adulterated pellets in one component and regular pellets in the alternate component, the first six (Phase 5a) and last five (Phase 5b) session rates for the mult VI 30 VI 30-sec schedule in Phase 5, and the mult VI 30 VI 30-sec schedule rates in Phase 6, with sucrose pellets as reinforcers in one component and regular pellets in the alternate component.

The upper and lower left portions of Fig. 1 show that the lesioned animals averaged above baseline rates (baseline indicated by the dashed horizontal line) on each day of the Phase 2 mult VI ext schedule, whereas the control group's rate of responding remained at baseline levels. In Phases 1 through 3, the light-on state was associated with the VI 30-sec schedule. In Phase 2, light-off was associated with extinction, and in Phase 3, light-off was associated with VI $30 \mathrm{sec}$.

As can be seen in Fig. 1, the grouped data (Phase 3a) reveal negative behavioral contrast for the $\mathrm{VMH}$-lesioned animals; accompanying an increase in response rate in the changed (light-off) component is a decrease in responding in the unchanged (light-on) component. Figures 2 and 3 contain the individual data for the VMH-lesioned animals for Phases 1 through 6, and it can be seen that positive behavioral contrast was demonstrated by all four lesioned animals on the Phase 2 mult VI ext schedule, and that negative behavioral contrast occurred for three of the four lesioned animals in Phase 3 (Ss 25, 27, and 29). S 36 demonstrated induction in Phase 3, insofar as his rate of response increased in the unchanged component when response rate increased in the changed component.

Figures 4 and 5 contain the individual data for the control animals for Phases 1 through 6 (with the 
exception of No. 28, for whom only the first three phases are shown, as the animal was eliminated in Phase 4 due to excessive variability in response rate). It can be seen in these figures that only two control animals showed increases in responding above baseline levels in Phase 2, and one of these Ss (No. 37) showed a decrease in rate below baseline levels following Session 4. None of the control animals displayed negative behavioral contrast in Phase 3 (mult VI VI).

Thus, the experimental hypothesis that animals with lesions in the ventromedial nucleus of the hypothalamus would show greater positive behavioral contrast effects than controls resulting from a reduction in reinforcement density in one component of a multiple schedule is supported by the results of Phase 2. The results of Phase 3, that three of the four lesioned animals displayed negative behavioral contrast effects as a result of an increase in reinforcement density in a changed component of a multiple schedule whereas none of the control animals did the same, also support the contention that VMH-lesioned animals are more responsive to changes in reinforcement contingencies than are controls.

\section{Reinforcement Quality Shift 1}

Table 3 contains a summary of the results for the Phase 4 mult VI 30 VI 30 -sec schedule shift, with adulterated pellets in one component and regular pellets in the alternate component. The mean preshift baseline rate (Phase 3b) for the experimental group was 33.6 responses/min and for the control group was 24.2 responses/min, the difference not being significant $[\mathrm{U}(4,4)=3.0, \mathrm{p}>.05]$. As can be seen in Figs. 2 and 3, the elevated rates in the Phase 2 VI component for the

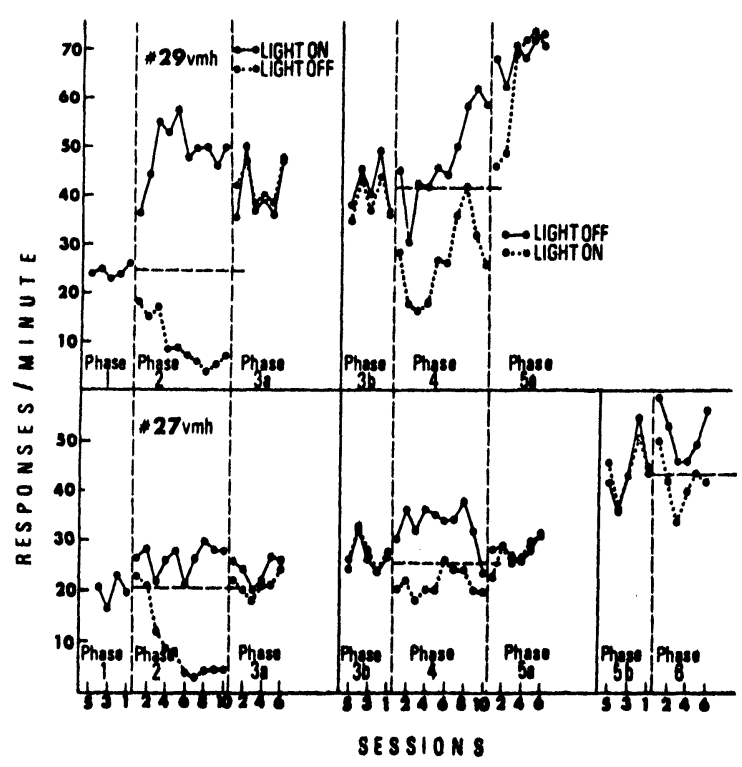

Fig. 3. Response rates for VMH-lesioned Animals 29 (upper graph) and 27 (lower graph) for Phases 1 through 6.

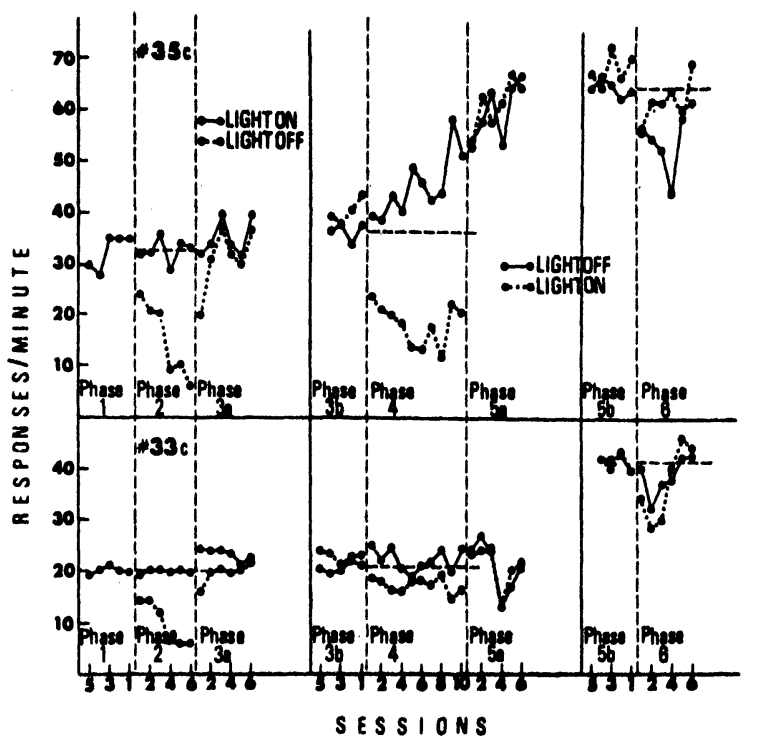

Fig. 4. Response rates for Control Animals 35 (upper graph) and 33 (lower graph) for Phases 1 through 6.

lesioned animals remained elevated above baseline in Phase 3.

It is clear from Figs. 2 through 5 that the control animals displayed greater positive behavioral contrast effects in Phase 4 than did the VMH-lesioned animals. The mean percent changes in rate over sessions in Phase 4 was +11.1 responses for the experimental group and +26.9 responses for the control group $(p<.05)$.

If daily session rates are treated as dependent measures, the hypothesis that animals with VMH lesions would display greater positive behavioral contrast than controls in response to adulterated pellets is not supported by the results of Phase 4. The failure to

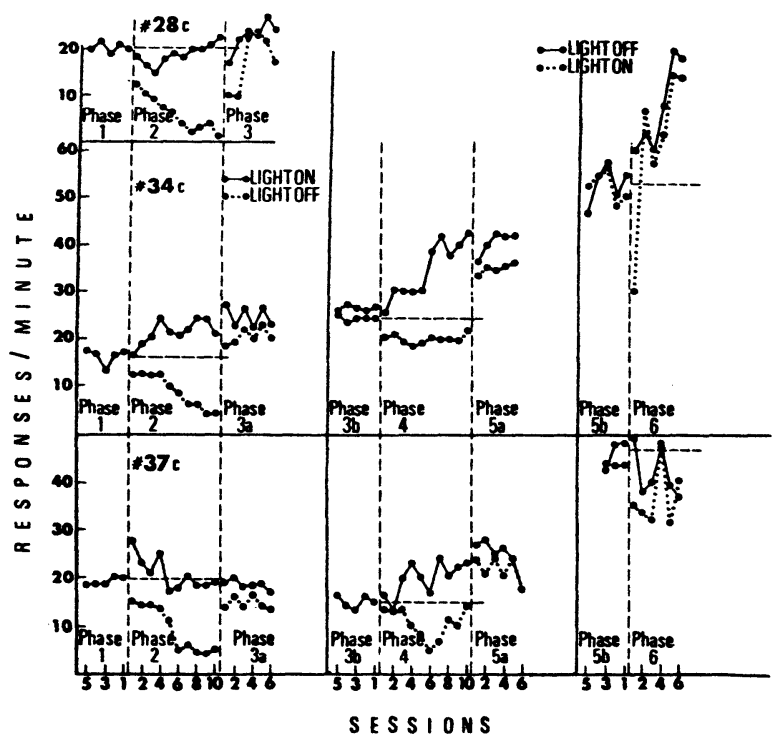

Fig. 5. Response rates for Control Animals 28 (upper graph), for Phases 1 through 3, 34 (middle graph), and 37 (lower graph), for Phases 1 through 6. 
Table 3

Summary of Results for Phase 4 (Quality Shift 1)

\begin{tabular}{cccccc}
\hline & Condition & $\begin{array}{c}\text { Preshift } \\
\text { Baseline } \\
\text { (Responses/ } \\
\text { Minute) }\end{array}$ & $\begin{array}{c}\text { Percent } \\
\text { Change in } \\
\text { Rate Over } \\
\text { Sessions }\end{array}$ & $\begin{array}{c}\text { Percent Change } \\
\text { in Rate First } \\
\text { 10 Min of } \\
\text { "Reg" Comp }\end{array}$ & $\begin{array}{c}\text { Percent Change } \\
\text { in Rate Last } \\
\text { 10 Min of } \\
\text { "Reg" Comp }\end{array}$ \\
\hline 33 & $\mathrm{C}$ & 20.6 & +6.8 & +10.7 & -2.9 \\
34 & $\mathrm{C}$ & 24.3 & +43.2 & +56.0 & +34.6 \\
35 & $\mathrm{C}$ & 36.8 & +24.0 & +43.5 & +26.4 \\
37 & $\mathrm{C}$ & $\overline{\mathrm{X}}=24.2$ & $\overline{\mathrm{X}}=+26.9$ & +38.7 & +20.0 \\
25 & $\mathrm{VMH}$ & 32.8 & +3.0 & $\overline{\mathrm{X}}=+37.2$ & $\overline{\mathrm{X}}=+19.5$ \\
27 & VMH & 25.6 & +29.3 & +29.6 & -12.5 \\
29 & VMH & 41.8 & +20.1 & +39.8 & +19.9 \\
36 & VMH & 34.1 & -7.9 & +50.2 & -9.6 \\
& & $\overline{\mathrm{X}}=33.6$ & $\overline{\mathrm{X}}=+11.1$ & $\overline{\mathrm{X}}=+32.3$ & $\overline{\mathrm{X}}=-7.3$ \\
\hline
\end{tabular}

support this hypothesis may be partially due to the large decrements in response rate within sessions that occurred for both groups, though more pronounced in the lesioned group.

In order to examine changes in response rate within sessions, mean changes in response rate for the first and last $10 \mathrm{~min}$ of the VI component during which regular food pellets served as reinforcers (VI reg) were averaged for representative Days 3, 6, and 9 for each animal in Phase 4, and compared to baseline rates of responding. It can be seen in Table 3 that the percent decrease in response rate within sessions relative to baseline is much greater for the lesioned animals than for the controls. If only the first $10 \mathrm{~min}$ of the VI-reg component are examined, the percent increase in rate over baseline was +32.3 for the lesioned animals and +37.2 for the controls ( $p>.05$ ). If the last $10 \mathrm{~min}$ of the same VI component are examined, the percent change in rate for the lesioned group (again compared to baseline) was -7.3 responses, and for the control group was +19.5 responses $(p<.05)$. Thus, there was a much greater within-session decrease in rate for the VMH-lesioned animals than for the controls, supporting the general hypothesis of relatively greater responsiveness to schedule manipulations in lesioned animals compared to controls.

Table 4

Summary of Results for Phase 6 (Quality Shift 2)

\begin{tabular}{lccc}
\hline S & Condition & $\begin{array}{c}\text { Preshift } \\
\text { Baseline } \\
\text { (Responses/ } \\
\text { Minute) }\end{array}$ & $\begin{array}{c}\text { Percent } \\
\text { Change } \\
\text { in Rate } \\
\text { VI Reg }\end{array}$ \\
\hline 33 & C & 41.7 & -7.2 \\
34 & $\mathrm{C}$ & 53.0 & +31.0 \\
35 & $\mathrm{C}$ & 65.0 & -14.6 \\
37 & $\mathrm{C}$ & 46.5 & -9.7 \\
& & $\overline{\mathrm{X}}=51.5$ & $\overline{\mathrm{X}}=-0.13$ \\
25 & VMH & 52.8 & -3.9 \\
27 & VMH & 43.7 & +17.8 \\
36 & VMH & 46.8 & +0.64 \\
& & $\overline{\mathrm{X}}=47.9$ & $\overline{\mathrm{X}}=+4.85$ \\
\hline
\end{tabular}

No differences were found between groups in percent decrease in rate relative to baseline over sessions in the Phase 4 component associated with adulterated pellets (VI adult). As can be seen in Fig. 1, the response rate decreased below baseline for both groups on the VI-adult component and remained fairly stable over sessions. The mean percentage decrease below baseline on session components involving the adulterated pellets were 36.2 and 38.6 for the lesioned and control animals, respectively. Though the number of adulterated pellets consumed was not systematically examined, informal observations showed that all animals ate almost all pellets for sessions observed.

\section{Reinforcement Quality Shift 2}

The upper and lower right-hand portions of Fig. 1 contain the results for both groups in Phase $5 \mathrm{~b}$ (baseline) and Phase 6 (mult VI 30 VI 30 with regular pellets in one component and sucrose pellets in the alternate component). Figure 1 demonstrates that both groups showed a mean decrease in response rate below baseline for the first four sessions in the sucrose component. The rate of response for the control group in the sucrose component increased over sessions to above baseline levels, whereas the VMH-lesioned group's rate gradually decreased over sessions until Session 5 . The individual data for Phase 6 can be seen in Figs. 2 through 5, and are summarized in Table 4.

The results of Phase 6 were unexpected insofar as the response rate for all but two animals decreased below baseline in the sucrose component of the multiple schedule. One control (No. 34) and one lesioned animal (No.27) showed elevated rates of response in the sucrose component for all six sessions in Phase 6. As can be seen in Table 4, the mean percentage changes in rate over sessions on the VI-reg component in Phase 6 were $+4.85 \%$ and $-0.13 \%$ for the lesioned and control groups, respectively $(p>.05)$. The mean percentage changes on the VI-sucrose component were $-5.35 \%$ and $-3.9 \%$ for the lesioned and control groups, respectively $(\mathrm{p}>.05)$.

Within-session changes in rate were examined for both 
groups for both components in Phase 6. The VMH-lesioned animals again demonstrated greater decrements in rate within the VI-reg component than the controls on both days observed $(p<.05)$. No significant changes in rate occurred for either group on the VI-sucrose component.

\section{DISCUSSION}

The results of the present experiment demonstrate that animals with lesions in the ventromedial nucleus of the hypothalamus show greater positive behavioral contrast than normals in a VI component of a multiple schedule when that component is alternated with extinction. In addition, three of the four lesioned animals displayed negative contrast effects on the mult VI VI schedule which followed the mult VI ext schedule, whereas none of the control animals displayed negative contrast. These two findings strongly support the hypothesis that VMH-lesioned animals are more responsive to changes in reinforcement density than are controls.

The results of the present study systematically replicated the findings of Jaffe and Caplan (1971) that rats with ventromedial hypothalamic lesions displayed marked contrast effects following shifts in reinforcement density on two fixed-interval schedules. The control animals in that study did not display contrast effects. Both studies taken together support the contention that VMH lesions increase the extent and/or probability of behavioral and incentive contrast when density of reinforcement is manipulated.

It is not clear why pronounced contrast effects were not reliably obtained in all control animals as is typically found in pigeons on multiple schedules when response rate is decreased in one component by decreasing reinforcement density in that component (Reynolds, 1961; Terrace, 1966). There are relatively few studies of behavioral contrast which use the rat as S. Pear and Wilkie (1969) used a modified multiple schedule in which the onset of the VI component was not signaled by a discriminative stimulus. They found that contrast "generally occurred" when extinction was used in the changed component. In a later study, Pear and Wilkie (1971), using parameters similar to those in the present study, found that two out of five normal rats exposed to a mult VI ext schedule following exposure to a VI 30-sec schedule showed large contrast effects in the unchanged component, two animals showed slight increases in rate over baseline, and one animal showed a decrease in rate. Freeman (1971), using a response-independent reinforcement paradigm, found no positive contrast in rats using a mult VI ext schedule. Thus, positive behavioral contrast apparently is less likely to occur in normal rats than in normal pigeons on similar multiple schedules. Pear and Gollub (personal communications) and pilot data for the present experiment suggested that the length of the components constituting the multiple schedule may be a crucial factor in determining the occurrence of contrast.

The hypothesis that animals with lesions in the ventromedial nucleus would show greater positive behavioral contrast effects than controls resulting from a reduction in the quality of reinforcement in one component of a multiple schedule was not supported by the results of Phase 4. As is shown in Table 3, the two groups did not differ in the percent increase in rate over baseline during the first 10 min of the VI-reg component in Phase 4, but the large within-session decrement in rate for the VMH-lesioned group resulted in a lower total percent increase in rate for the sessions as a whole.

That the VMH-lesioned animals did not show greater percentage increases in rate relative to baseline in the unchanged VI component than the controls may be attributed to their previous experience in Phase 2. It has been shown that extended discrimination training on mult VI ext with rats (Pear \& Wilkie, 1971) or pigeons (Terrace, 1966) leads to a gradual decrease in positive behavioral contrast, until the rate in the unchanged component approximates the initial baseline rate. Although the number of sessions of mult VI ext was relatively small in the present study, and despite the fact that none of the lesioned animals demonstrated decreasing rates in the last few mult VI ext sessions, it is possible that having displayed contrast under one set of conditions decreased the probability or extent of contrast under a different set of conditions.

Regarding the quality shifts in Phases 5 and 6, it can be seen in Fig. 1 that there was a large increase in response rate in the control group during the course of Phase 5 (baseline mult VI VI). The increase in rate was probably due to the addition into the logic program of a component which prevented the reinforcement of barholding. Barholding behavior was not observed prior to this phase, however.

The main result of the Phase 6 multiple schedule with sucrose pellets in one component was that the sucrose pellets were less palatable than the regular pellets for both groups insofar as the rates decrease in the VI-sucrose component below baseline levels. Singh (1970) found that both male and female VMH-lesioned rats consumed significantly less sucrose pellets under a CRF schedule than did controls. It may be that $100 \%$ sucrose pellets are less palatable to rats than regular pellets. Because of this unexpected finding, no conclusions can be made regarding the responsiveness of VMH-lesioned rats to a highly palatable reinforcer compared to controls.

The results of the present study contradict the hypothesis that VMH-lesioned animals are not as "willing" to work for food as controls or that they will not work "as hard" for food compared to controls. This hypothesis is specifically contradicted by the following results: (1) There was no change in baseline rate for the lesioned animals on the VI 30-sec schedule (Phase 1) following recovery from the operation; (2) the 
VMH-lesioned animals barpressed more frequently than the controls on the VI component of the mult VI ext schedule in Phase 2: (3) the absolute rates of responding were greater for the lesioned animals than for the controls on the VI-reg component in Phase 4, and (4) there were no differences between groups in percent decrease in rate relative to baseline in the extinction component of Phase 2. in the VI-adult component of Phase 4. or in either component of the Phase 6 mult VI VI schedule. The data of the present study suggest that animals with lesions in the ventromedial nucleus may respond at a higher rate than do controls, depending upon factors related to the contingencies of reinforcement operating in the experimental situation, specifically reinforcer density.

The results of the present study also suggest that the claim of decreased hunger motivation (or increased finickiness) in the $\mathrm{VMH}$-lesioned animal may be too general. It appears that the reinforcement contingencies in earlier studies (e.g., Teitelbaum, 1955) may have been responsible for the lesioned animals' responding with lower rates than controls.

\section{REFERENCES}

Corbit. J. D. Hyperphagic hyperreactivity to adulteration of drinking water with quinine $\mathrm{HCL}$. Journal of Comparative \& Phy siological Psychology. 1965. 60, 123-124.

Corbit. J. D.. \& Stellar, E. Palatability, food intake, and obesity in normal and hyperphagic rats. Journal of Comparative \& Phy siological Psychology, 1964, 58, 63-67.

deGroot. J. The rat forebrain in stereotaxic coordinates. Amsterdam: Noorde-Hollanshe Uitgevers Maatschappij, 1959.

Dunham. P. J. Contrasted conditions of reinforcement: A selective critique. Psychological Bulletin, 1968, 69, 295-315.

Epstein. A. N., \& Teitelbaum, P. Regulation of food intake in the absence of taste, smell, and other oropharyngeal sensations. Journal of Comparative \& Physiological Psychology. 1962, 55. 753-759.

Freeman. B. J. The role of response-independent reinforcement in producing behavioral contrast effects in the rat. Learning \& Motivation, 1971. 2, 138-147.

Graff. H.. \& Stellar. E. Hyperphagia, obesity and finickiness. Journal of Comparative \& Physiological Psychology, 1962, 55. 418-424.
Grossman. S. P. The VMH: A center for affective reactions. satiety. or both? Physiology \& Behavior, 1966, 1, 1-9.

Grossman. S. P. A textbook of physiological psychologr. New York: Wiley. 1967.

Jaffe. M. L.. \& Caplan, M. The effect of switched reinforcement schedules on response rates of rats with ventromedial lesions. Paper presented at the meeting of the Eastern Psychological Association. New York. April 1971.

Konig. J. F. R.. \& Klippel, R. A. The rat brain: A stercotaxic atlas. Baltimore: Williams \& Wilkens. 1963.

Miller, N. E.. Bailey, C. J., \& Stevenson. J. A. F. Decreased hunger but increased food intake resulting from hypothalamic lesions. Science, 1950, 112, 256-259.

Pear. J. J.. \& Wilkie. D. M. Stimulus changes as a discriminative event in a semi-multiple schedule. Proceedings. 77 th Annual Convention. APA. 1969, 797-798.

Pear. J. J.. \& Wilkie. D. M. Contrast and induction in rats on multiple schedules. Journal of the Experimental Analysis of Behavior. 1971, 15, 289-296.

Revnolds. G. S. Behavioral contrast. Journal of the Experimental Analysis of Behavior, 1961, 4. 57-71.

Sclafani. A. Neural pathways involved in the ventromedial hypothalamic lesion syndrome in the rat. Journal of Comparative \& Physiological Psychology. 1971. 77. 70-96.

Sechzer, J. A., Turner, S. G.. \& Liebelt, R. A. Motivation and learning in mice after goldthioglucose induced hypothalamic lesions. Psychonomic Science, 1966. 4. 259-260.

Singh, D. Sex differences in obesity and food-directed activity in normal and hyperphagic rats. Psychonomic Science, 1970. 21. 306-308.

Teitelbaum. P. Sensory control of hypothalamic hyperphagia. Journal of Comparative \& Physiological Psychology. 1955. 48, 158-163.

Teitelbaum. P. Random and food-directed activity in hyperphagic and normal rats. Journal of Comparative \& Pinysiological Psychofogy, 1957, 50. 486-490.

Teitelbaum, P., \& Epstein, A. N. The role of taste and smell in the regulation of food and water intake. In Proceedings of the First International Symposium on Olfaction and Taste. London: Pergamon Press, 1963.

Terrace, H. S. Stimulus control. In W. K. Honig (Ed.), Operant behavior: Areas of research and application. New York: Appleton-Century-Crofts, 1966.

Turner. S. G.. Sechzer, J. A., \& Liebelt. R. A. Sensitivity to electric shock after ventromedial hypothalamic lesions. Experimental Neurology, 1967, 19. 236-244.

(Received for publication January 20, 1973; accepted March 15. 1973.) 